\title{
Breeding cooperation: cultural evolution in an intergenerational public goods experiment
}

\author{
Vicken Hillis $^{1}$ and Mark Lubell ${ }^{1}$
}

\begin{abstract}
The transmission of cooperative norms among individuals across generations plays a key role in our ability to successfully manage social-ecological systems in changing environments. Here, we use an intergenerational public goods experiment combining both cooperative advice and in-game communication in order to examine the transmission of cooperative norms across generations of experimental participants. We show that cooperative intergenerational advice has a positive impact on both (i) contributions by individuals in a subsequent generation and (ii) the cooperative content of communication among individuals in a subsequent generation. The impact of cooperative intergenerational advice is most pronounced at the beginning of the subsequent generation. The impact of in-game communication, on the other hand, is relatively consistent over the course of the experiment. Sessions combining advice and communication have the highest levels of cooperation overall. Our findings highlight the potential contributions of intergenerational experiments to research in social-ecological systems more generally.
\end{abstract}

Key Words: cultural evolution; intergenerational experiment; public goods game; social-ecological systems

\section{INTRODUCTION}

In this paper, we report the results of a public goods experiment that uses an intergenerational structure to mirror real-world settings where cooperative institutions evolve over time via cultural transmission. Specifically, we find that it is possible to breed cooperation by selectively exposing a second generation of subjects to cooperative advice about game play from an initial generation. Intergenerational experiments of this type provide a useful experimental approach for testing hypotheses about how cultural evolutionary processes affect cooperation in socialecological systems (SES).

Individuals rely heavily on social learning, or the transmission of behaviors among individuals via teaching or imitation, in order to shape their own decision making (Boyd and Richerson 1985). Such transmission of behaviors among individuals creates a process of cultural evolution (Boyd and Richerson 1985, CavalliSforza and Feldman 1981). Social learning can take various forms, including vertical (learn from one's parents), oblique (learn from other individuals in an older generation) or horizontal (learn from one's peers). Theoretical work in cultural evolution predicts a substantial reliance on social learning under moderate levels of environmental variability (Henrich and McElreath 2003), and more recent research has turned to comparing the conditions that favor vertical, oblique, and horizontal social learning. McElreath and Strimling (2008) demonstrated that vertical transmission is preferred when the environment is stable or selection is strong, and that oblique transmission is important when the environment changes quickly, because oblique transmission responds to environmental change more rapidly. Aunger (2000) examined food taboos and found that vertical transmission is most important early in the life course whereas horizontal transmission is important later. In general, however, there is no consensus, either from theoretical modeling or empirical research, about which type of social learning (vertical vs. horizontal) will dominate under different conditions. Furthermore, relatively little work has explored vertical and horizontal social learning in the context of cooperative dilemmas, and SES more generally.
Cultural evolution has important consequences for the dynamics and resilience of SES, where over time stakeholders build institutions to support cooperative outcomes. Many enduring common-pool resource institutions studied by Ostrom (1990) and others operated over multiple human generations and depended on the establishment of traditions of cooperation passed down successfully across these generations. For example, patrilineally transmitted territorial rights contributed to the sustainability of lobster harvests in Maine during the late $20^{\text {th }}$ century (Acheson 1975). Cooperative traditions are also critical to the success of institutions in more modern settings, such as the spread of collaborative governance in environmental and other policy arenas. A common theme from the literature on collaborative governance is that collaborative policies are more likely to succeed when they are built on a foundation of preexisting trust and social capital (Sabatier et al. 2005, Ansell and Gash 2008).

In general, a major challenge for the sustainability of SES is maintaining cooperation in populations made up of constantly changing individuals. Even in the short term, actors in governance institutions will often move around, as occurs when a government agency rotates personnel across regions. In the long term, as institutions evolve across human lifespans, institutions and cultural norms must be transmitted to new generations. Overcoming this challenge requires the cultural transmission of cooperative norms among individuals. Interest in the relevance of social learning to outcomes for SES management is increasing (Keen et al. 2005, Pahl-Wostl et al. 2007). For example, Berkes (2009) nominated social learning as a key component of the successful comanagement of natural resources. Napier et al. (2005) found that the most successful comanagement regimes of subsistence fisheries in South Africa have an important time element and that the longer they have been operating, the more successful they are.

The real-world examples discussed above involve complicated processes of social learning over long periods of time, including participatory and iterative processes that create feedbacks and reinforcement loops. Although our experimental design does not 
capture all of the richness of these real-world systems, it does capture a key feature important to many real-world SES - that experienced individuals communicate information to naive individuals about how to behave and that this process can play an important role in shaping cooperative outcomes.

Although it is difficult to stake out new territory in the mature and vast literature on experimental social dilemmas, intergenerational experiments are a relatively new and sparse subset of this literature. Some initial studies have been conducted using a variety of types of social dilemmas and methods of cultural transmission. One series of experiments suggests that whereas cultural transmission has an extremely strong influence on behavior in later generations, there are some interesting asymmetric effects, such as perpetuating unequal divisions of gains from cooperation (Schotter and Sopher 2003), decreasing levels of trust among senders in a trust game but increasing levels of trustworthiness among receivers (Schotter and Sopher 2006), and considerations of fairness being more important for receivers than senders in an ultimatum game (Schotter and Sopher 2007). Using a public goods game with punishment, Gürerk (2012) finds that providing subjects with a social history of a previous experiment increases social efficiency in two ways: first, because subjects reduce their reluctance to initially choose the punishment option (with respect to a baseline experiment when subjects are not provided with a social history) and second, because subjects reach a cooperative equilibrium more quickly and spend less on punishments than they otherwise would. In another experiment incorporating recommendations, Croson and Marks (2001) find a positive effect of recommendations only when individuals value the public good heterogeneously but not homogeneously. A common theme from all of the above experiments is that the intergenerational structure provides novel insights that would otherwise be difficult to uncover.

The experiment conducted in the past most similar to our own comes from Chaudhuri et al. (2006), who conducted a public goods game in which subjects received advice from individuals in previous experiments. The advice took three different forms: private knowledge, in which the advice left by one player was available to only a single successor in the subsequent generation; public knowledge, in which advice left by all players in the initial generation was made available to all players in the subsequent generation; or common knowledge, in which the advice from all players in the previous generation was read aloud to all players in the subsequent generation. Chaudhuri et al. (2006) found that only advice in the form of common knowledge increased contributions to the public good compared with a baseline treatment with no advice. This positive effect of advice in the form of common knowledge was maintained across three generations of subjects. Importantly, Chaudhuri et al. (2006) also demonstrated that when advice was transmitted as common knowledge, the content of the advice evolved to be very cooperative.

Our experiment builds on this finding by selecting cooperative advice and examining the influence of that cooperative advice in the presence of in-game communication. Our intent is to examine the maximum potential for the transmission of cooperative norms, analogous to the artificial selection of traits in domesticated plants and animals. A major advantage of intergenerational experiments is the potential to investigate different individual and contextual factors that may strengthen or weaken different cultural transmission processes. In this case, we are purposely enhancing the influence of vertical transmission; in theory this could be mimicked in the field by selecting successful groups (or individuals within groups) to advise new groups about managing SES. In effect, we shortcut to the cooperative advice that Chaudhuri et al. (2006) find evolves naturally, in order to examine the effect of advice in the presence of in-game communication.

A large number of social dilemma experiments have previously examined the importance of communication among players. This work has proceeded from early demonstrations of the effectiveness of face-to-face communication (Isaac and Walker 1988) to reviews demonstrating the robustness of this claim (e.g., Sally 1995) in a variety of settings and communication media, and more recently, attempts to uncover the mechanisms underlying the generally positive effect of communication. The intergenerational structure of our experiment allows us to examine how the cooperative content of communication changes over time in response to cooperative advice.

Our experiment examines the effect of cooperative advice and communication, both on their own and in concert. We find that cooperative advice has a substantial positive effect on contributions to the public goods game, but that this effect is more pronounced at the beginning of a game and declines over time. We also find that communication has a positive effect on cooperation, but that there are no positive (or negative) synergies between communication and advice. Finally, we find that advice increases the cooperative content of communication among individuals within a game, but that this effect is relatively mild and again is most pronounced at the beginning of a game and declines over time.

\section{METHODS}

Undergraduate students in groups ranging from four to nine individuals $(n)$ played a public goods game. In each round $(t)$, each individual $(i)$ could contribute between 0 and 10 tokens to a common fund. Contributions $(C)$ to the common fund were doubled and returned to all members of the group regardless of how much, if anything, they contributed themselves. Hence, as with traditional public goods experiments, the Nash equilibrium in a single round of the game is to contribute nothing and free ride on the contributions of other players. Group welfare, however, is maximized when all subjects contribute maximally to the common fund. Considering a contribution to the common fund by player $i$ in round $t$ as $C_{i t}$, the payoff $(\pi)$ for each subject in a given round was:

$$
\pi_{i t}=10-C_{i t}+(2 / n) \sum_{i=1}^{n} C_{i t} ; t=1, \ldots, 10 .
$$

We used a $2 \times 2$ experimental design that combined two different treatments (presence or absence of in-game communication and between-generation advice) in the standard public goods structure described above. In treatments that included in-game communication, subjects could send one anonymous message to all other individuals in each round. In treatments that included between-generation advice, subjects received advice from previous participants about how to play the game. This advice was generated via a single, open-ended, anonymous question 
posed to all subjects at the end of a previous session. We selected the set of most cooperative advice from a single first-generation group (first-generation groups did not receive any advice themselves) to pass on to all second-generation sessions. This filtering of advice serves as a cost-effective mechanism to efficiently produce cooperative advice (as occurred naturally in Chaudhuri et al.'s (2006) experiment) without using deception. We provide the details for this process below. Table 1 summarizes the number of groups and subjects in each treatment corresponding to the combinations of between-game advice and in-game communication.

Table 1. Treatments, number of sessions, and total number of individuals in each treatment. The majority of sessions consisted of groups of five individuals, although this ranged from four to nine. We used all treatments in the analysis of contribution decisions. We used only treatments involving communication (rows 1 and 3 ) in the analysis of communication content.

\begin{tabular}{lcc}
\hline \hline Treatment & Sessions & Individuals \\
\hline No communication; no advice (NC-G1) & 9 & 47 \\
Ingame communication only (C-G1) & 8 & 41 \\
Between-game advice only (NC-G2) & 10 & 46 \\
Both communication and advice (C-G2) & 12 & 57 \\
\hline
\end{tabular}

In a given round, subjects could contribute up to 10 tokens to the common fund. Subjects accumulated or lost tokens over the course of the experiment and were paid in cash at the end of the game in proportion to the amount of tokens they had accumulated, plus a $\$ 5$ show-up payment. Subjects earned between $\$ 10-20$, with an approximate average of $\$ 15$.

All experiments were run via a computer network using the experimental software Gameweb (http://sourceforge.net/projects/ gameweb/). Visual examples of all stages explained below are included in Appendix 1. Subjects initially read instructions informing them of the payoff structure and details of the game operation. Subjects were informed that interactions in the game and payment amounts would be anonymous. To help subjects understand the game, they were provided with an introductory screen in which they could test how different levels of contribution amounts would affect payoff distributions. However, we did not require them to correctly calculate payoffs for different gameplay scenarios before proceeding.

After experimenting with contribution and payoff amounts on this introductory screen, subjects received advice from a previous generation. The advice was presented with minimal explanation using the following statement: "Previous participants in this experiment left the following advice for you based on their experiences." In order to minimize differences between groups receiving advice and groups not receiving advice, those not receiving advice also saw the screen about advice, with the following message in place of advice, "No advice supplied because you are the first set of individuals to participate in this experiment."

Advice was solicited from all individuals in all treatments at the end of the experiment using the following solicitation: "The main part of the experiment is now over, and you have gained a bit of experience and learned how best to play this game. Please take a moment to write a paragraph to the next group of participants, telling them any suggestions you now have about how to play the game. You may be as detailed as you wish in your recommendations. As always, your identity will never be displayed alongside what you write here." Subjects were not compensated directly for providing this advice, nor were their final payoffs impacted by the performance of groups receiving the advice they presented. Although compensating past subjects is a common procedure in intergenerational experiments, here we were not interested in testing the effects of the incentive, and compensating past subjects for the performance of future generations would have been very difficult without either resorting to deception or conditionally compensating only the small subset of subjects whose advice was actually linked to future generations.

In each round of the experiment, subjects initially contributed some amount to the common fund, and then were able to view (anonymously) the per-round contributions, per-round payoffs, and cumulative payoffs for each individual in the game. After viewing these, subjects were able to send a message to other participants (if they were in a session that included within-game communication), and then could choose to read the messages of other (anonymous) players. Each game lasted 20 rounds, although subjects were told only that the experiment lasted for an unspecified, set number of rounds, in order to reduce potential end-game effects.

We classified each message produced by within-game communication as to whether or not it included an explicit exhortation to the other players to contribute the full amount of ten tokens. Researchers use a variety of methods to classify language, including self-classification (e.g., Schotter and Sopher 2007), content analysis (e.g., Krippendorff 2004), and coordination games (e.g., Houser and Xiao 2011). Although each method has its merits, we chose content analysis because it combines relative cost effectiveness (compared with an incentivized coordination game) with a procedure for validation (as opposed to self-classification). Following Lombard et al. (2002), we used the following procedure to produce a reliable set of classified messages. First, we jointly developed a coding framework via discussions about the important concepts we wanted to identify in the messages (we identified a number of concepts, but use only one [full cooperation] in this paper). Next, the first author used the coding instrument to code all messages as to whether or not they included an explicit exhortation to contribute the full amount of 10 tokens. For example, "hey...10 in solidarity, right community?" was coded as an exhortation to contribute fully whereas "sweet baby jesus" was not. Finally, we chose two separate random samples that were coded independently, each by a single different reliability coder (these recoded samples were each separately compared with the original codings prepared by the first author). The reliability coders were graduate and undergraduate students affiliated with the authors' research lab but blind to the specific hypotheses of this experiment. They were not compensated financially for their coding work, which took about 1-2 hours, including a brief training period and the coding itself. The reliability samples consisted of 99 and 141 messages selected randomly from the full population of messages. We calculated Krippendorrf's alpha for each reliability sample $(0.88$ and 0.78$)$, considered good and adequate, respectively. 
Fig. 1. Distributions of contributions to the public good in each of the four treatments (by row) for all 20 rounds (left-most column of panels), split up by rounds 1-10 (middle column of panels), and by rounds 11-20 (rightmost column of panels). Red vertical line in each panel indicates the mean contribution amount for that treatment and round combination. The distributions indicate a tendency to contribute either all or nothing to the public good. Means indicate increasing contributions due to both communication and advice, with the effect of advice most pronounced in early rounds, and the effect of communication relatively consistent across all rounds.

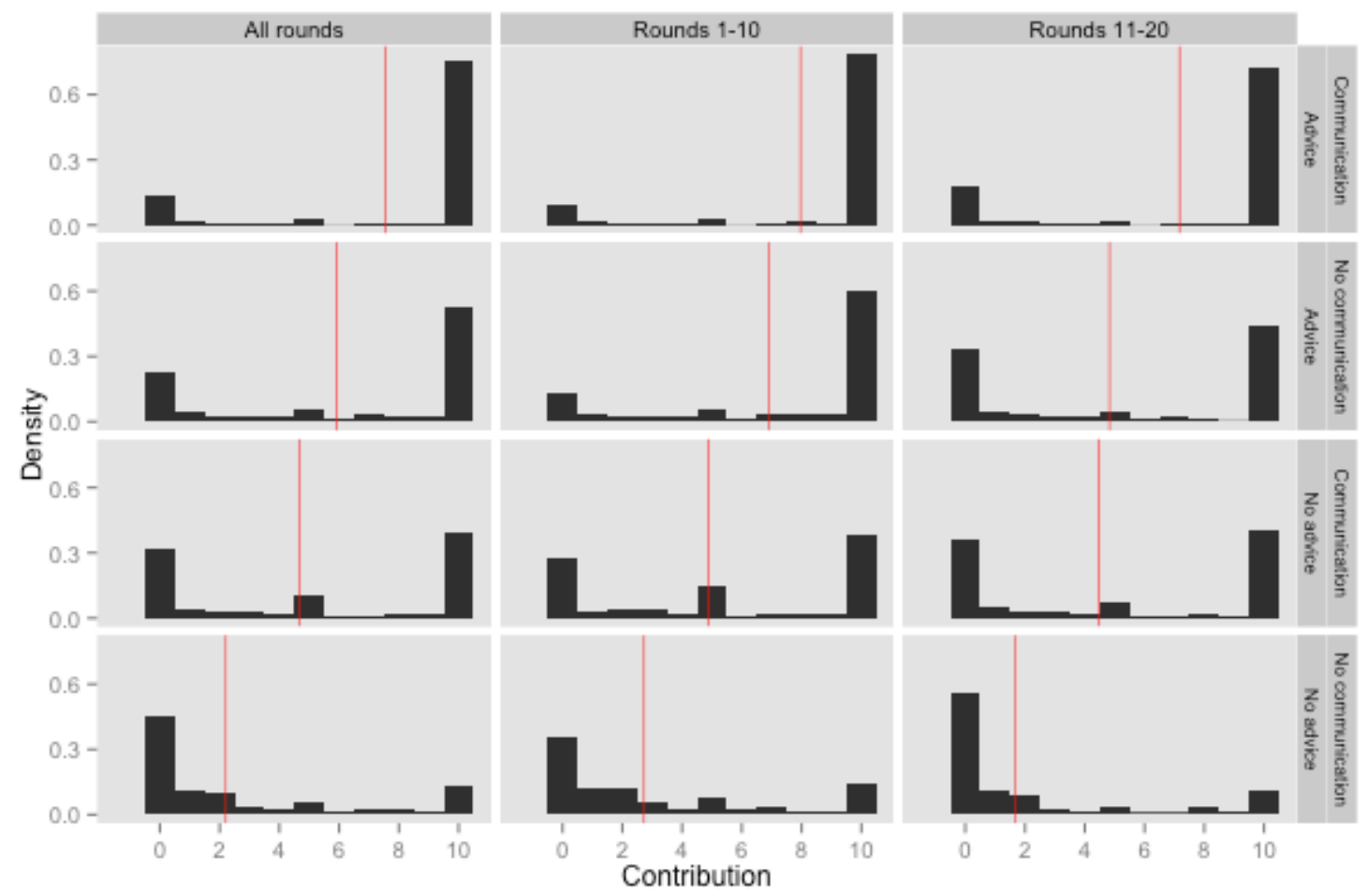

We used a similar, but less formal, procedure to classify the most cooperative set of advice from a single first-generation session to pass on to all subsequent generations. We chose to classify each piece of advice into three possible categories: suggesting cooperation, suggesting defection, and neither. Although this categorization ignores a good deal of nuanced meaning in the advice, it was adequate for the simple purpose of selecting the most cooperative advice. The first author coded each piece of advice into these three categories, and subsequently trained two undergraduate assistants on the procedure and had them do the same. The undergraduate assistants were involved with the research project and not blind to the research hypotheses. We did not calculate a quantitative measure of reliability for these codings, and although there were certainly some differences in the classifications, a single consensus set of most-cooperative advice emerged. We reviewed the classifications with the second author, who confirmed the use of that set. The specific pieces of advice (four) transmitted to second-generation sessions are presented here:

1. "It's so much better when you do all 10."

2. "If EVERYONE gives 10 the system will work and you will walk out with as much $\$ \$$ as possible"
3. "Well we tried to keep everyone putting the amount of money in and it worked but at round 20 someone cheated and put in zero. I would say to just stay faithful and put in 10. Although calling people immature for abusing the system works sometimes. lol have fun."

4. "All of you guys should put 10 every time to get the most amount of money and the same amount split between you guys! Trust each other!"

\section{HYPOTHESES}

Our experiment addressed the following specific hypotheses:

1. Cooperative advice increases the level of cooperation in subsequent generations.

If vertical or oblique social learning is important, then we should see a positive effect of cooperative advice on cooperation. As discussed above, a broad body of theoretical work in cultural evolution (Boyd and Richerson 1985, Henrich and McElreath 2003) leads us to predict that individuals will attend to information from individuals in a previous generation. Here, we examine a particular form of vertical or oblique social learning when the strength of transmission is strong (i.e., the cooperative content of advice is high). 
2. Cooperative advice changes the content of in-game communication, increasing the proportion of in-game messages sent that support cooperation.

If indeed cooperative advice has a positive effect on cooperation, identifying the mechanism by which this occurs is important. We know that in-game communication often has a positive effect on cooperation (Sally 1995) and that the content of a message can influence a receiver's response to it (Parks et al. 1996). Here, we examine whether advice has a positive influence on cooperation because it helps make communication more cooperative and thus more effective.

3. Cooperative advice and in-game communication complement each other, such that there is a positive synergy between the two (i.e., in statistical terms, a positive interaction).

Following logically from hypothesis 2, if advice makes communication more cooperative, then there may be a positive synergy between the two. More generally, although both the effect of communication and the effect of advice have been examined independently, no study has combined the two to examine the effect of each in the presence of the other, and cultural evolutionary theory leaves open the question as to which we expect to influence cooperation in a social dilemma. Furthermore, Chaudhuri et al.'s (2006) finding that the positive effect of advice declines over time suggests that there may be room for a positive interaction between advice and communication (advice generates high levels of cooperation early in the game, and communication maintains cooperation at these levels over the course of the game).

\section{RESULTS}

We start by examining the distributions of contributions in the four treatments (Fig. 1). The distributions are usually bimodal; most participants appear to be deciding to contribute either the full amount or nothing at all, with a moderate amount contributing half in certain treatments, and a scattering of other partial contributions. The treatment with the lowest amount of mean contribution overall (2.71) is that with no advice and no communication, followed by communication but no advice (5.18), advice but no communication (6.43), and finally both advice and communication (8.06). Corresponding to the changes in averages across treatments is a decrease in the proportion of subjects contributing nothing and an increase in the proportion of subjects contributing maximally.

This pattern of differences in contributions across treatments largely holds when examining the first ten rounds and the last ten rounds separately, with the key difference that, in the initial ten rounds, the difference between communication without advice and advice without communication is more pronounced and, in the final ten rounds, that difference is attenuated. This suggests that the effect of advice is greatest early in the game and that the effect of communication is relatively important later in the game. Indeed, comparison of average contributions in the two treatments reveals that contributions decline over time in the presence of advice without communication (7.39 in rounds $1-10$ and 5.33 in rounds $11-20)$, whereas contributions are relatively flat in the presence of communication without advice (5.39 in rounds $1-10$ and 4.96 in rounds $11-20$ ).
To further examine the differences across treatments over time, in Fig. 2, we plot mean contributions for each treatment in each round. As seen in the figure, contributions in the treatment with no communication and no advice (NC-G1) start at about half of the maximum possible and trend down over time to less than a quarter at the end of the experiment. In the communication-only treatment $(\mathrm{C}-\mathrm{G} 1)$, cooperation again starts off roughly around half of the total possible and is then maintained at more or less the same level for the duration of the game. In the advice-only treatment (NC-G2), cooperation starts much higher, at almost the maximum possible amount, and cooperation levels then decline to roughly half the total possible over the course of the game. Finally, when between-game advice and in-game communication are combined (C-G2), cooperation levels begin near the maximum possible amount and are maintained at almost that level for the duration of the game.

Fig. 2. Mean contribution to the public good across all individuals in all sessions by round for each of the four treatment combinations. NC-G1: Baseline treatment with no communication and no advice (first generation). C-G1: Communication only treatment with no advice (first generation). NC-G2: Advice only (second generation) treatment with no communication. C-G2: Advice (second generation) and communication both.

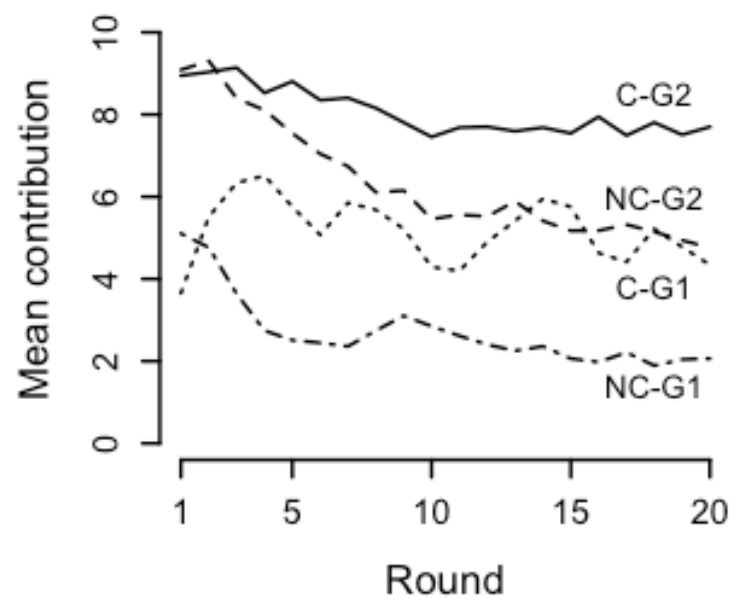

In the aggregate, these trends imply that the primary effect of advice is to increase the initial contribution amount of groups in the second generation, and that the primary effect of communication is to maintain contributions at whatever level they start at, whether this is middling in groups that didn't receive advice, or very high in groups that did.

To complement this visual and descriptive examination, we assess the statistical influence of our predictor variables (dummy variables for advice and communication, the round in which the contribution was made, and the size of the group playing the game, as well as relevant interactions among these predictors) on our outcome variable, contribution amounts. Group sizes ranged between four and nine individuals (although a substantial majority of sessions had five individuals, and only two sessions had more than six individuals; one with seven individuals and another with nine individuals). Thus, controlling for the size of 
Table 2. Parameter estimates, standard errors (in parentheses), and associated $p$ values (* indicates $p<0.05$ ) from a series of mixedeffect Tobit regressions that account for the bounded nature of the dependent variable, contributions to the public good. Each model (by column) includes a random intercept for players, accounting for the clustering of contributions made by a single participant and fixed effects for the predictor variables (by row). Interactions between predictor variables included in the models are indicated by an " $\mathrm{x}$ ".

\begin{tabular}{|c|c|c|c|c|c|}
\hline & Model 1 & Model 2 & Model 3 & Model 4 & Model 5 \\
\hline Advice & $12.076 *(1.698)$ & $12.077 *(2.402)$ & $12.007 *(1.694)$ & $15.359 *(1.835)$ & $15.356 *(1.828)$ \\
\hline Communication & $5.426 *(1.617)$ & $5.427 *(2.331)$ & $2.071(1.714)$ & $5.471 *(1.630)$ & $1.962(1.728)$ \\
\hline Round & $-0.383 *(0.029)$ & $-0.383 *(0.029)$ & $-0.537 *(0.041)$ & $-0.260 *(0.036)$ & $-0.413 *(0.045)$ \\
\hline Groupsize & $-1.355(0.733)$ & $-1.355(0.738)$ & $-1.345(0.731)$ & $-1.290(0.738)$ & $-1.267(0.737)$ \\
\hline Advice $\mathrm{x}$ Communication & & $-0.002(3.243)$ & & & \\
\hline Round x Advice & & & & $-0.306^{*}(0.058)$ & $-0.315^{*}(0.057)$ \\
\hline Round x Communication & & & $0.318 *(0.056)$ & & $0.325^{*}(0.056)$ \\
\hline Constant & $11.506 *(4.264)$ & $11.505 *(4.446)$ & $13.044 *(4.266)$ & $9.907 *(4.305)$ & $11.413 *(4.305)$ \\
\hline Log likelihood & -4803.639 & -4803.639 & -4787.670 & -4789.500 & -4772.485 \\
\hline Wald $\chi^{2}$ & $239.61 *$ & $239.61 *$ & $265.54 *$ & $258.87^{*}$ & $287.37^{*}$ \\
\hline Sigma epsilon & 7.329 & 7.329 & 7.260 & 7.249 & 7.177 \\
\hline Number uncensored & 972 & 972 & 972 & 972 & 972 \\
\hline Number lower censored & 1030 & 1030 & 1030 & 1030 & 1030 \\
\hline Number upper censored & 1758 & 1758 & 1758 & 1758 & 1758 \\
\hline Number of players & 191 & 191 & 191 & 191 & 191 \\
\hline
\end{tabular}

the group is important because the marginal benefit of contributions declines as group size increases (Walker and Isaac 1988). We did attempt to interact the treatment variables with group size but were not able to estimate this effect accurately due to relatively limited observations of small and large group sizes.

We fit a set of mixed-effect (also called random effect, hierarchical, or multilevel) Tobit regressions with a random intercept for players, accounting for the clustering of the contributions made by a particular player, and fixed effects for the various predictors and interactions. We also fit a series of other models that account for the clustering of contributions within players, and players within games, in other ways. These include Tobit regressions with robust standard errors clustered at either the level of the game or player, Tobit regressions (bounded only from below, with only main effects estimated) with two-way robust standard errors clustered by both game and player, and ordered logistic regressions with random intercepts for both game and player. All of these other analyses (not reported but available from the authors) produce results that are qualitatively the same as those presented here.

Table 2 reports parameter estimates, standard errors, and associated $p$ values for these Tobit regressions for the contribution of player $i$ in round $t$. We report results from models that include all main effects and various combinations of the interactions that were estimated accurately (treatment variables with each other and with round). First, estimates for advice are consistently positive across all models, confirming that subjects receiving cooperative advice contribute more to the public good. This confirms our first hypothesis. Second, communication has a positive effect on advice that is consistent across all models, confirming that subjects contribute more when they can send each other messages during the game. Third, the effect of round on contributions is consistently negative, confirming that contributions overall decline over the course of the experiment. Fourth, the interaction effect between advice and communication is negligible, indicating the effect of advice does not depend on whether or not subjects can communicate (and vice versa). Thus, with respect to our third hypothesis, in the statistical analysis, we don't find any evidence of either a positive or negative synergy between advice and communication. Finally, the interaction between round and advice is negative, but the interaction between round and communication is positive. These interactions confirm that the positive effect of cooperative advice is strongest early in games, whereas the positive effect of communication is most pronounced later on in games.

In order to address our second hypothesis about the influence of advice on the cooperative content of communication, we first plot the proportion of total messages in a given round, across all players and sessions, that explicitly exhort other players to contribute fully (Fig. 3) for both the first and second generations (G1 and G2, respectively). As seen in the figure, the proportion of messages exhorting full cooperation is higher in the second generation than in the first, but only for the first few rounds of the game.

Fig. 3. Percentage of possible messages that explicitly exhorted full cooperation, by round and treatment, considering only the two treatments in which subjects could communicate. G1: first generation. G2: second generation.

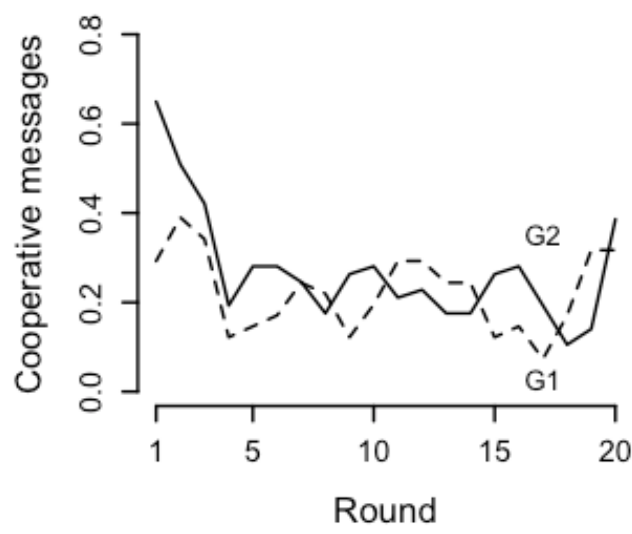


Table 3. Parameter estimates, standard errors (in parentheses), and associated $p$ values (* indicates $p<0.05$ ) from a series of mixedeffect logistic regressions. The dependent variable in each model (by column) is whether or not a message exhorted other players to contribute fully to the public good. Each model includes a random intercept for players, accounting for the panel nature of the data, and fixed effects for the predictor variables (by row). Interactions between predictor variables are indicated by an " $\mathrm{x}$ ".

\begin{tabular}{lcccc}
\hline \hline & Model 1 & Model 2 & Model 3 & Model 4 \\
\hline Advice & $0.363(0.245)$ & $0.941^{*}(0.318)$ & $-6.437^{*}(1.898)$ & $-5.881^{*}(1.912)$ \\
Round & $-0.054^{*}(0.010)$ & $-0.018(0.015)$ & $-0.054^{*}(0.010)$ & $-0.019(0.016)$ \\
Groupsize & $0.078(0.194)$ & $0.085(0.195)$ & $-0.719^{*}(0.289)$ & $-0.713^{*} 0.290$ \\
Advice x Round & & $-0.059^{*}(0.020)$ & & $-0.059^{*}(0.020)$ \\
Advicex Groupsize & & & $1.350^{*}(0.375)$ & $1.354^{*}(0.376)$ \\
Constant & $-1.363(1.022)$ & $-1.750(1.036)$ & $2.771(1.503)$ & $2.387(1.510)$ \\
sigma_epsilon & 0.973 & 0.986 & 0.822 & 0.833 \\
Log likelihood & -1015.0 & -1010.8 & -1008.7 & -1004.6 \\
AIC & 2039.9 & 2033.6 & 2029.5 & 2023.2 \\
BIC & 2067.8 & 2067.1 & 2063.0 & 2062.3 \\
Observations & 1960 & 1960 & 960 & 1960 \\
Number of players & 98 & 98 & 98 & 98 \\
Number of games & 20 & 20 & 20 & 20 \\
\hline
\end{tabular}

To complement this visual examination, we fit a set of mixedeffect logistic regressions again with a random intercept for players, accounting for the clustering of messages sent by a particular player. The outcome variable in these regressions is the cooperative content of message $i(0=$ not cooperative; $1=$ cooperative) delivered in round $t$, and the predictor variables are modeled as fixed effects and include whether or not advice was available in that session, the round in which the message was sent, the size of the group in that session, and interactions between group size and round as well as group size and the treatment dummy variable for advice. We also fit models with random intercepts for both players and games, accounting for both levels of clustering in the data. These models (not presented here but available from the authors) produce results that are qualitatively the same as those presented here. For consistency with the cooperation analysis, we report results from the models with only a random intercept for players.

Table 3 reports parameter estimates, standard errors, and associated $p$ values for these logistic regressions for the cooperative content of message $i$ in round $t$. As seen in the table, estimates for advice in models 1 and 2 are consistently positive, indicating that individuals are more likely to exhort other players to cooperate fully when they have received cooperative advice. This positive effect of advice is mediated by the negative estimate for round and the negative interaction between advice and round. These estimates indicate that the cooperative content of messages decreases as an experiment progresses and that the positive influence of advice on the cooperative content of messages also declines over time. Finally, in models that include an interaction between group size and advice, we uncover a negative effect of group size and a positive interaction between group size and advice (the sign of the estimate for advice also becomes negative).

Given the inconsistency in sign and magnitude of the estimates across models, and the nonlinearity and hierarchical nature of the models, we calculate and report predicted probabilities to help interpret the parameter estimates in the table. In the calculation of predicted probabilities, we use model 4 , which has the lowest AIC score and accounts for about $95 \%$ of the AIC weight for all models fit. The predicted probabilities of sending a cooperative message confirm that the overall effect of receiving cooperative advice is still slightly positive (moving from $24.3 \%$ for those not receiving advice to $28.8 \%$ for those who do, for an average player, holding other predictors constant) but that there is a strong interaction between group size and advice, such that advice has a negative impact for group sizes of four (the minimum group size for the communication games) and a positive impact for group sizes of six (maximum group size for communication games). We consider this interaction to be somewhat speculative, however (even if statistically significant), because observations of group sizes of four and six are very limited in the data.

Although we think the finding is interesting because it hints that intergenerational transmission of advice plays an important role in promoting cooperative communication in larger groups (where collective action problems tend to be most acute), we don't explore this result further in the discussion, due to the limited observations of group size. To check the robustness of the positive effect of advice on the cooperative content of communication, we also fit the same set of models (excluding any models with group size or associated interactions) with a truncated data set including only groups with five individuals. These models (not shown, but available from the authors) replicate our primary qualitative findings, namely, that cooperative advice promotes cooperative messaging, but that this effect is attenuated over time.

\section{DISCUSSION}

The experiment reported here demonstrates that it is possible to increase cooperation by selectively exposing later generations to advice that recommends cooperation. Why, then, does advice have such a strong effect on cooperation? Although further experiments are needed to untangle some of the possible causal mechanisms driving the effect of advice and examining it over multiple generations, we highlight some plausible explanations here.

One explanation is that players are simply responding to the information content contained in the advice provided. This is analogous to acting on instructions about how best to deal with 
a confusing situation. An alternative explanation that considers the strategic incentives of the social dilemma is that players change their expectations of the type of player they expect to encounter in the experiment. Most players in social dilemma experiments are conditional cooperators and will cooperate as long as they are interacting with other subjects who are also cooperating (Fischbacher et al. 2001). Cooperative advice may thus serve to signal to players that they can expect to interact with other cooperators in the experiment. This is consistent with the notion that the effect of advice is strongest at the beginning of the game, before the information content of the advice is diluted by the reality of behavior by other players in the experiment, particularly if other players defect. Chaudhuri and Paichayontvijit (2006) demonstrate that information about the presence of conditional cooperators in a game increases cooperation. Cooperative advice in our experiment may similarly be indicating to subjects the expected presence of conditional cooperators.

Another explanation for the effect of advice is that individuals may be using a conformist bias to guide their decision making in these dilemmas. Cultural evolutionary theory has shown that conforming to the majority behavior can be adaptive in a range of different conditions and that conformist transmission can help to stabilize cooperation (Henrich and Boyd 2001) and that cultural group selection can lead to the spread of prosocial behaviors among groups (Boyd and Richerson 2009). Thus, cultural evolution can play a substantial role in the development of institutions to solve collective action problems (Richerson and Henrich 2012). These results are consistent with previous experiments that have demonstrated a role for conformity in social dilemma experiments (Carpenter 2004, Bardsley and Sausgruber 2005). Although our experiment doesn't provide direct evidence of conformity, our results are consistent with the use of a conformist bias to guide decision making.

Another reason that advice may have a strong positive effect is that it comes from a group of impartial individuals (as opposed to the senders of in-game communication, who are involved in a strategic interaction with the receivers). Evidence from studies of the evolution of teaching (Kline 2014) indicates that pupils are, to some extent, skeptical of teachers. In our advice condition, senders (or "teachers") have no motivation to deceive receivers (or "pupils"). In the communication condition, senders have a potential interest in deceiving receivers, because of the potential increased payoff to themselves of motivating others to contribute more. This reasoning is consistent with our results and actually predicts that advice might have a greater positive impact on cooperation than in-game communication. An experiment that compares the effects of unselected advice with cooperation could test this prediction.

Of course, one of the reasons we see such a strong effect of intergenerational advice is because we purposely selected the most cooperative advice to transmit to the subsequent generation, in order to examine the maximum possible cooperative impact of intergenerational advice. This type of experimental design is analogous to early experiments in artificial selection designed to breed varieties of corn with both very high and very low oil content (Dudley and Lambert 2004). The results here are thus representative of the maximum cooperative potential of intergenerational advice. This suggests an obvious extension to our experiment involving either advice evolving freely over time or selecting for the most negative advice with respect to cooperative content. This would facilitate a fair comparison of the strength of the effect of advice and cooperation, and thus, vertical and horizontal social learning, as well as an examination of potential asymmetries between the evolution of cooperation and defection via intergenerational cultural transmission.

An additional caveat to our main finding, the importance of advice, is that at least some part of our result may be generated by what are called experimenter demand effects (Zizzo 2010) or changes in behavior on the part of subjects as a result of cues about appropriate behavior in the experiment. In our case, experimenter demand effects may indeed be playing a role if subjects interpret advice as a cue from experimenters as to the appropriate or expected behavior and are motivated to meet experimenter expectations. Having said that, various features of our design work to inhibit the importance of an experimenter demand effect, including not using a senior PI as the experiment administrator and limiting social interactions between subjects and the administrator to those required to conduct the experiment. Furthermore, the between-subjects nature of the design minimizes subject perception that advice and communication are the key variables of interest in the study, thus obscuring the objectives of the experiment and minimizing subjects' ability to construe experimenter demand.

The results reported here open up a host of interesting questions about intergenerational transmission and cooperation that deserve serious exploration. Further experiments might examine what types of experimental treatments influence the strength of intergenerational cultural transmission and the persistence of cooperative norms over time. For example, Schotter and Sopher (2003) run their experiments for long enough to observe punctuated equilibria, where some groups exhibit much lower levels of cooperation. We suspect a similar phenomenon would occur if we conducted additional generations in the setting we use because there is always a probability of one or more defectors joining the experiment and ignoring the advice of previous generations. The question, then, would be to examine the relative lengths of the periods of cooperation and defection in the longer term.

Other obvious extensions involve increasing the degree of realism in various ways with respect to real-world SES. This might include modifying the structure of the social dilemma (e.g., using a common-pool resource game), incorporating levels of spatial and temporal realism that better represent SES (Janssen et al. 2010), or using overlapping generations instead of the nonoverlapping generations used here.

Another important area for extension is to understand how intergenerational advice interacts with other institutional arrangements such as the potential to punish defectors and reward cooperators (Fehr and Gächter 2000, Sefton and Steinberg 1996). Does intergenerational advice increase the effectiveness of other institutions, such as punishment and rewarding, in the same way that it appears to improve communication?

\section{CONCLUSION}

Understanding the impact of intergenerational advice on cooperative behavior in a range of settings has important real- 
world implications for institutions in cooperative dilemmas. Our experimental manipulations here are analogous to resource management decisions that expose new generations of people to particular types of information and behaviors from previous generations. This, of course, happens all the time in the real world as people channel the information available to their successors in various human enterprises. Pinpointing the mechanisms through which cultural transmission most effectively promotes cooperation in the lab should help inform those choices in the field. In real SES, cultural evolutionary forces will lead to complex interactions among institutions and behavior that will influence system resilience over time.

Responses to this article can be read online at: http://www.ecologyandsociety.org/issues/responses. $\mathrm{php} / 7424$

\section{Acknowledgments:}

We thank Peter J. Richerson and Richard McElreath for comments on an earlier version of the manuscript, Richard McElreath and Carl McCabe for software development, and Matt Berke, Morgan Chow, Matthew Hoffman, and Isaac Walrath for assistance collecting and coding data.

\section{LITERATURE CITED}

Acheson, J. M. 1975. The lobster fiefs: economic and ecological effects of territoriality in the Maine lobster industry. Human Ecology 3(3):183-207.

Ansell, C., and A. Gash. 2008. Collaborative governance in theory and practice. Journal of Public Administration Research and Theory 18(4):543-571.

Aunger, R. 2000. The life history of culture learning in a face-toface society. Ethos 28(3):1-38.

Bardsley, N., and R. Sausgruber. 2005. Conformity and reciprocity in public good provision. Journal of Economic Psychology 26:664-681.

Berkes, F. 2009. Evolution of co-management: role of knowledge generation, bridging organizations and social learning. Journal of Environmental Management 90:1692-1702.

Boyd, R., and P. J. Richerson. 1985. Culture and the evolutionary process. The University of Chicago Press, Chicago, Illinois, USA.

Boyd, R., and P. J. Richerson. 2009. Culture and the evolution of human cooperation. Philosophical Transactions of the Royal Society B 364(1533):3281-3288. http://dx.doi.org/10.1098/ rstb.2009.0134

Carpenter, J. P. 2004. When in Rome: conformity and the provision of public goods. Journal of Socio-Economics 33:395408.

Cavalli-Sforza, L., and M. Feldman. 1981. Cultural transmission and evolution: a quantitative approach. Princeton University Press, Princeton, New Jersey, USA.
Chaudhuri, A., S. Graziano, and P. Maitra. 2006. Social learning and norms in a public goods experiment with inter-generational advice. The Review of Economic Studies 73(2):357-380. http://dx. doi.org/10.2139/ssrn.580481

Chaudhuri, A., and T. Paichayontvijit. 2006. Conditional cooperation and voluntary contributions to a public good. Economics Bulletin 38):1-14.

Croson, R. and M. Marks. 2001. The effect of recommended contributions in the voluntary provision of public goods. Economic Inquiry 39(2):238-249.

Dudley, J., and R. Lambert. 2004. 100 generations of selection for oil and protein in corn. Plant Breeding Reviews 24(1):79-110.

Fehr, E., and S. Gächter. 2000. Cooperation and punishment in public goods experiments. The American Economic Review 90 (4):980-994.

Fischbacher, U., S. Gächter, and E. Fehr. 2001. Are people conditionally cooperative? Evidence from a public goods experiment. Economics Letters 71(3):397-404.

Gürerk, Ö. 2012. Social learning increases the acceptance and the efficiency of punishment institutions in social dilemmas. Journal Of Economic Psychology 34:229-239.

Henrich, J., and R. Boyd. 2001. Why people punish defectors: weak conformist transmission can stabilize costly enforcement of norms in cooperative dilemmas. Journal of Theoretical Biology 208:79-89.

Henrich, J., and R. McElreath. 2003. The evolution of cultural evolution. Evolutionary Anthropology 12:123-135.

Houser, D., and E. Xiao. 2011. Classification of natural language messages using a coordination game. Experimental Economics 14:1-14.

Isaac, R. M., and J. M. Walker. 1988. Communication and freeriding behavior: the voluntary contribution mechanism. Economic Inquiry 26(4):585-608.

Janssen, M. A., R. Holahan, A. Lee, and E. Ostrom. 2010. Lab experiments for the study of social-ecological systems. Science 328(5978):613-617.

Keen, M., V. A. Brown, and R. Dyball. 2005. Social learning in environmental management: towards a sustainable future. Earthscan, New York, New York, USA.

Kline, M. A. 2014. How to learn about teaching: an evolutionary framework for the study of teaching behavior in humans and other animals. Behavioral and Brain Sciences: online early.

Krippendorff, K. 2013. Content analysis: an introduction to its methodology. Third edition, Sage Publications, Inc., Thousand Oaks, California, USA.

Lombard, M., J. Snyder-Duch, and C. C. Bracken. 2002. Content analysis in mass communication: assessment and reporting of interceder reliability. Human Communication Research 28(4):587604.

McElreath, R., and P. Strimling. 2008. When natural selection favors imitation of parents. Current Anthropology 49(2):307-316. 
Napier, R. N., G. M. Branch, and J. M. Harris. 2005. Evaluating conditions for successful co-management of subsistence fisheries in KwaZulu-Natal, South Africa. Environmental Conservation 2:165-177.

Ostrom, E. 1990. Governing the commons: the evolution of institutions for collective action. Cambridge University Press, New York, New York, USA.

Pahl-Wostl, C., M. Craps, A. Dewulf, E. Mostert, D. Tabara, and T. Taillieu. 2007. Social learning and water resources management. Ecology and Society 12(2):5. [online] URL: http:// www.ecologyandsociety.org/vol12/iss2/art5/

Parks, C. D., R. F. Henager, and S. D. Scamahorn. 1996. Trust and reactions to messages of intent in social dilemmas. Journal of Conflict Resolution 40(1):134-151.

Richerson, P. J., and J. Henrich. 2012. Tribal social instincts and the cultural evolution of institutions to solve collective action problems. Cliodynamics: the Journal of Theoretical and Mathematical History 3(1):38-80. http://dx.doi.org/10.2139/ $\underline{\text { ssrn.1368756 }}$

Sabatier, P. A., W. Focht, M. Lubell, Z. Trachtenberg, A. Vedlitz, and M. Matlock. 2005. Swimming upstream: collaborative approaches to watershed management. MIT Press, Cambridge, Massachusetts, USA.

Sally, D. 1995. Conversation and cooperation in social dilemmas a meta-analysis of experiments from 1958 to 1992. Rationality and Society 7(1):58-92.

Schotter, A., and B. Sopher. 2003. Social learning and coordination conventions in intergenerational games: an experimental study. Journal of Political Economy 111(3):498-529.

Schotter, A., and B. Sopher. 2006. Trust and trustworthiness in games: an experimental study of intergenerational advice. Experimental Economics 9(2):123-145.

Schotter, A., and B. Sopher. 2007. Advice and behavior in intergenerational ultimatum games: an experimental approach. Games and Economic Behavior 58(2):365-393.

Sefton, M., and R. Steinberg. 1996. Reward structures in public good experiments. Journal of Public Economics 61(2):263-287.

Walker, J. M., and R. M. Isaac. 1988. Group size effects in public goods provision: the voluntary contributions mechanism. The Quarterly Journal of Economics 103(1):179-199.

Zizzo, D. J. 2010. Experimenter demand effects in economic experiments. Experimental Economics 13:75-98. 


\section{Appendix 1. Game instructions.}

Introduction, page 1.

Welcome.

In this experiment, you will be asked to make decisions about how to divide money between yourself and the other experiment participants. You will be paid in cash for your participation. Your decisions will affect not only how much money you earn, but also how much the other participants earn. Likewise, decisions made by the other participants will affect your earnings.

Please read the instructions carefully because the amount of money you earn depends on your understanding of the experiment. If you have any questions during the experiment, please silently raise your hand and the administrator will come to assist you.

Try to spend no more than a minute or so on each instructions page.

\section{Continue}


Introduction, page 2.

\section{Instructions}

In this experiment, each participant is given a stake of points $(50)$ in a private account. These points will be converted into real money at the end of the game with each point worth $\$ 0.05$. In each round of decision-making you and other participants are asked to contribute some of the points from your private accounts to a public account. You may contribute any amount, from zero to the maximum of 10 points (10 points equals $\$ 0.50)$. After all participants have made their decisions about donating to the public account, the administrator will multiply the contributions by 2 and equally distribute the amount among all participants regardless of how much each contributed.

Each round of this experiment will consist of a game like this, and your private account balance will continue to accumulate or decrease based on your net earnings in each round. The experiment will continue for a set number of rounds, and you can try to earn as much money as possible during those rounds.

\section{Test Out an Example of the Experimental Game}

To better understand how this experimental game works, you may adjust the Contribution Amounts below. For the example game of four players (including you), try to enact these four possible scenarios:

- Your highest individual earnings is realized when you contribute nothing but all other participants contribute everything.

- Your lowest indivdual earnings is realized when you contribute everything but no other participant contributes anything.

- The highest average earnings is realized when all participants contribute everything.

- The lowest average earnings is realized when all participants contribute nothing.

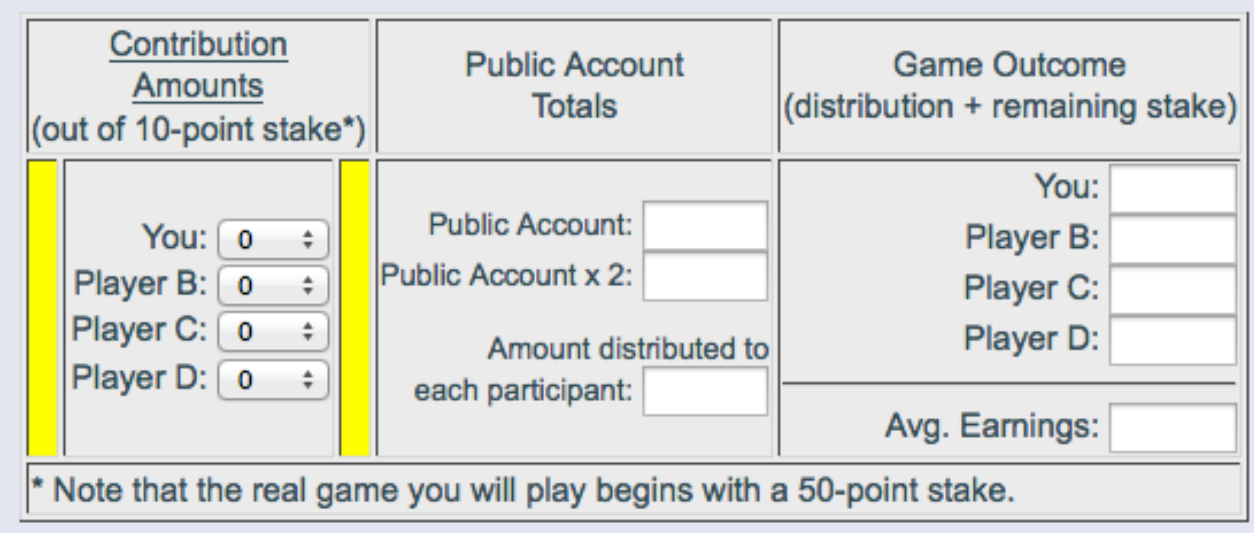

\section{Display of Information About Your Payoffs}

Some information in the game will be presented to you in the form of these mouse-over boxes. When you see these boxes, you may move your mouse pointer over the box to see the information that corresponds to the box's label. Try mousing over the following boxes to see how it works:

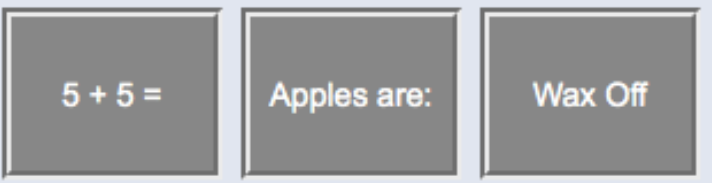

Continue 
Round:1, View messages/advice.

\section{View Advice from Participants in the Previous Game}

Previous participants in this experiment left the following advice for you based on their experiences.
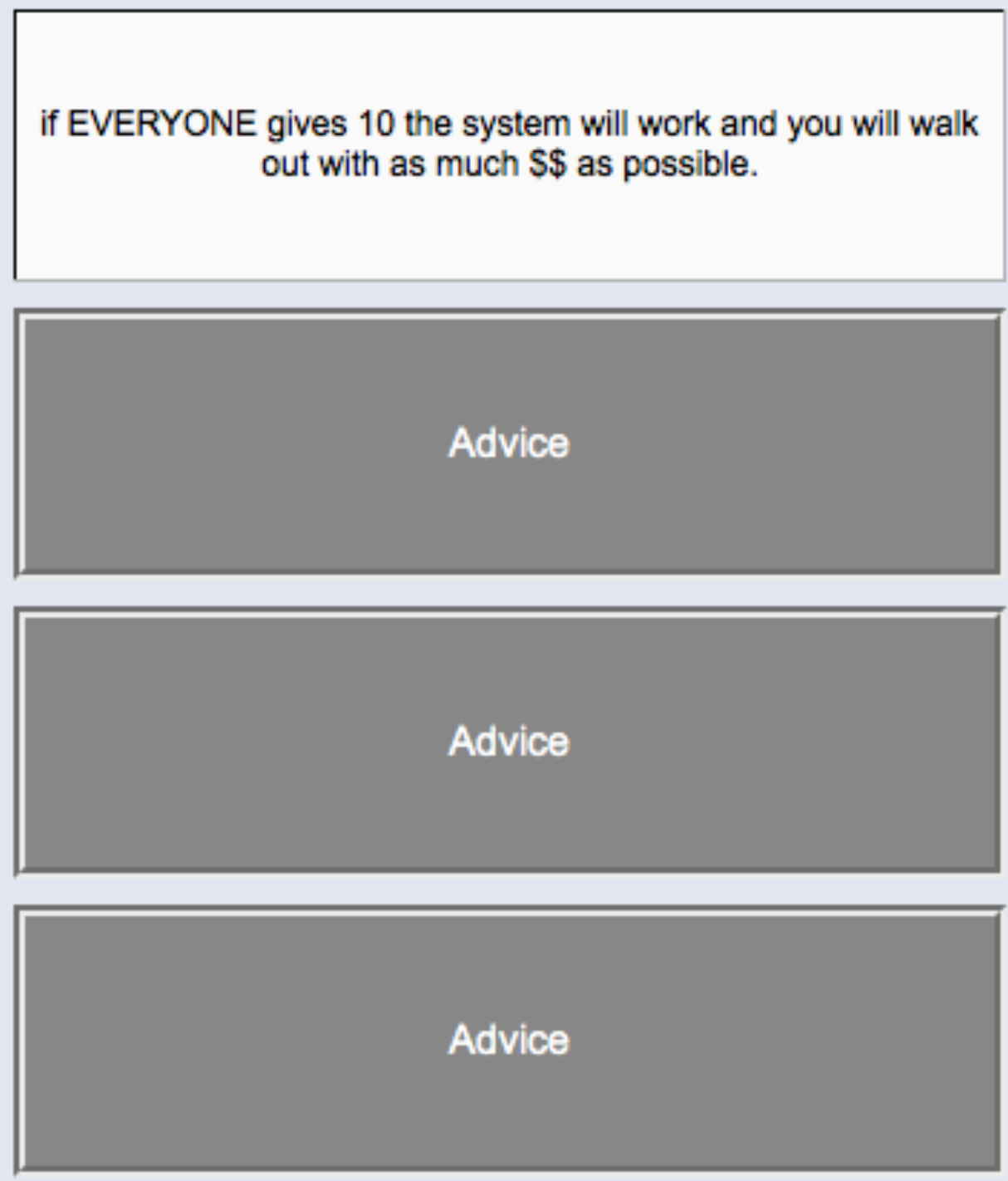

Submit 
Round:1, View messages/advice.

\section{View Advice from Participants in the Previous Game}

Previous participants in this experiment left the following advice for you based on their experiences.

No advice supplied because you are the first set of individuals to participate in this experiment.

\section{Advice}

Advice

Advice 
Round:1, Make your decisions.

\section{Decide on Your Contribution Amount}

Your Contribution: Please use the following input method to select how much you would like to contribute to the public account in this round. If you don't want to make a contribution, then select "0" (zero) from the drop-down list. 
Round:1, View results.

Below are the results of this round of the experiment. Move your mouse over any box to see the corresponding information. When you've seen as much as you're interested in, click the 'Submit' button to continue on to the next stage.

\section{Public Account Distribution:}

This is the amount each player received in this round after the public account was multiplied by 2 and equally divided among all players.

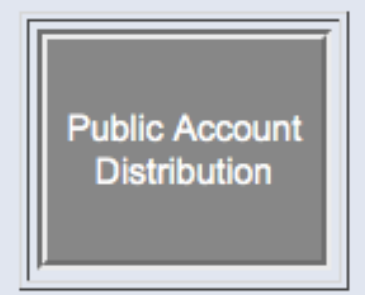

\section{Your Game Play and Changes to your Private Account Balance:}

These are the details of your experimental decisions. The "New Private Account Balance" includes the amount you earned in this round plus your initial endowment and the earnings you have from previous rounds.

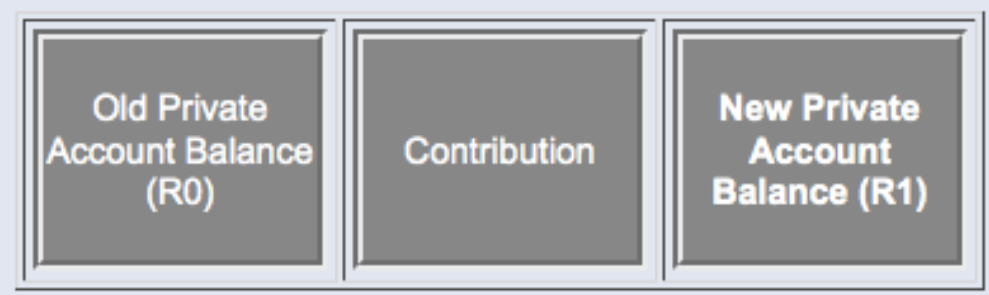

\section{Other Player's Game Play and Changes to Their Private Account Balances:}

For each other player, their contribution is listed above their new account balance. Again, the "New Private Account Balance" includes earnings from this round plus their initial endowment and earnings from previous rounds.

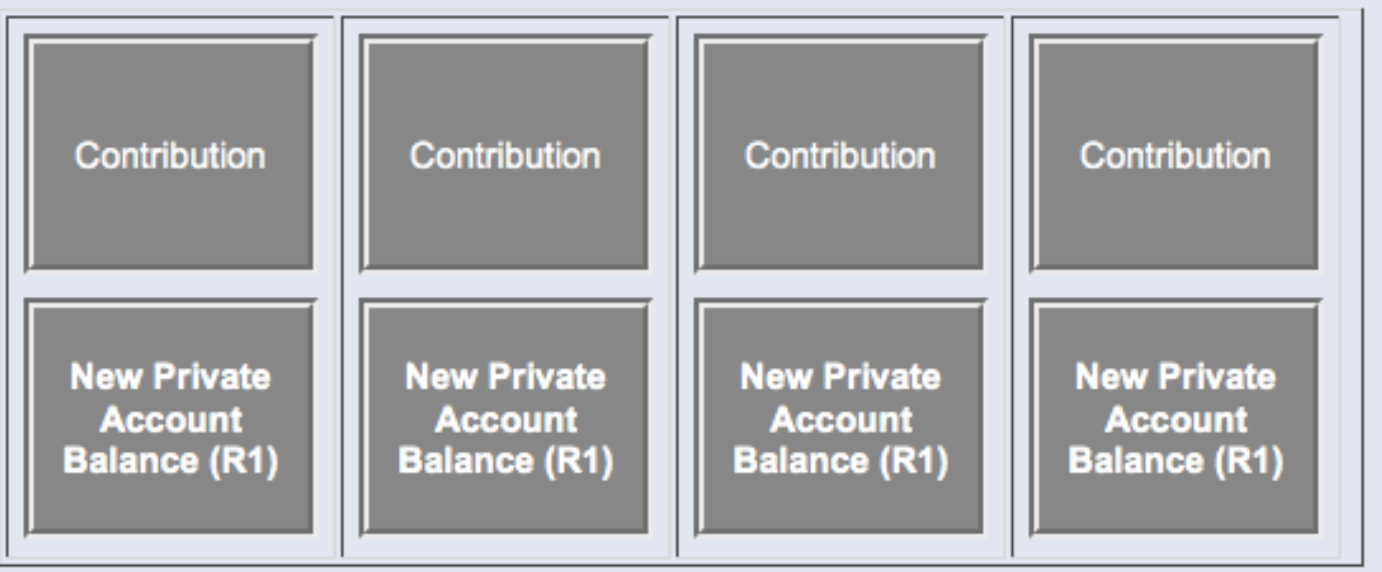


Round:1, Write messages/advice.

\section{Write a Message to the Other Participants}

Please use the following input box to write a message to the other participants in this game. This will be displayed together with messages from other participants before anyone makes decisions in the next round.

All of the other participants will be able to see your message, but the identity of the sender remains anonymous. You will also be able to see the messages from other participants, but you won't know their identity either. Even if you have no message, type "I have no message." so that everyone types something. 
Round:7, Write messages/advice.

\section{Leave Advice for Next Group of Participants}

The main part of the experiment is now over, and you have gained a bit of experience and learned how best to play this game. Please take a moment to write a paragraph to the next group of participants, telling them any suggestions you now have about how to play the game. You may be as detailed as you wish in your recommendations. As always, your identity will never be displayed alongside what write here. 